\title{
Amiloidosis primaria localizada en glande peneano. Caso clínico y revisión de la literatura
}

\author{
Domínguez Domínguez M, Valero Puerta JA, Jiménez Leiro JF, Martínez Ruiz R, \\ Medina Pérez M*.
}

Servicio de Urología. *Servicio de Anatomía Patológica. Hospital de la Merced. Osuna. Sevilla

Actas Urol Esp. 2007;31(2):168-171

\section{RESUMEN}

AMILOIDOSIS PRIMARIA LOCALIZADA EN GLANDE PENEANO. CASO CLÍNICO Y REVISIÓN DE LA LITERATURA

La amiloidosis primaria del tracto genitourinario es rara siendo aún más excepcional la afectación única del glande. Generalmente la clínica apunta al diagnóstico de carcinoma de pene y es el examen histológico el que indicará definitivamente el diagnóstico. La Biopsia de la lesión revela depósitos dérmicos de material amorfo eosinofílico. Se presenta el caso de un paciente con amiloidosis primaria en glande. Se realiza revisión de la literatura y se analizan las diferentes actitudes diagnósticas y terapéuticas. Éste es el séptimo caso de amiloidosis primaria localizada en glande descrito en la literatura.

Palabras claves: Amiloidosis. Glande. Pene. Uretra. Tracto urinario.

\section{ABSTRACT \\ PRIMARY LOCALIZED AMYLOIDOSIS OF GLANS PENIS. A NEW CASE AND REVIEW OF THE LITERATURE}

Primary localized amyloidosis urinary tract is rare. Localized amyloidosid only glands is a very rare entity. The clinical impression may resemble neoplastic disease but the diagnosis is confirmed by histochemical study. Biopsy of the lesion revealed dermal deposits of amorphous eosinophilic material. A case of patient with primary amyldosis of glands penis is reported. The literature is reviewed and the diagnostic and therapeutic options are discussed. This is the seventh reported case of localized amyloidosis of the glands penis.

Keywords: Amyloidosis. Glands. Penis. Urethra. Genitourinary.

$\mathrm{L}^{2}$ a amiloidosis se define como una serie de entidades clínicas y bioquímicas que se caracterizan por el depósito extracelular de la proteína fibrilar amiloide en una o más localizaciones del organismo. El amiloide es un material amorfo, eosinófilo, extracelular y de distribución ubicua. La tinción de Rojo Congo hace que presente una birrefrigencia verde exclusiva cuando los cortes histológicos se observan bajo luz polarizada $^{1,2}$. La amiloidosis puede ser primaria, asociada a mieloma múltiple, reactiva, heredo familiar (polineuropatía amiloidótica familiar), local, senil o por envejecimiento y amiloidosis relacionada con la hemodiálisis crónica. Las manifestaciones clinicas de amiloidosis dependen de la zona del organismo a la que afectan ${ }^{3}$.

El diagnóstico de amiloidosis se realiza mediante biopsia y estudio anatomopatológico y la demostración de amiloide en esta muestra mediante tinciones adecuadas. Los depósitos de amiloide se tiñen de color rosa con la tinción de hamatoxilina-eosina y presentan metacromasia con la tinción de violeta de genciana. Las muestras tisulares obtenidas deben teñirse con Rojo 
Congo y examinarse bajo luz polarizada para observar la típica birrefrigencia verde, éste es el procedimiento más útil para establecer la presencia de amiloide sistémico ${ }^{1}$.....

La presencia de amiloidosis en el aparato genitourinario, sin presencia de amiloidosis generalizada, ha sido documentada ${ }^{4-7}$. La localización en el pene es infrecuente y cuando se ve afectado suele tratarse de formas de amiloidosis uretral ${ }^{8}$. Sin embargo la afectación única del glande es excepcional $^{9-14}$.

El pronóstico de la enfermedad es muy variable y va a depender del tipo de amiloidosis y del órgano al que afecte. En el caso de la amiloidosis localizada, como es el caso que nos ocupa, será imprescindible realizar un estudio analítico y clínico adecuado para descartar otras asociaciones (Ej. Mieloma) o afectación de otros órganos (amiloidosis sistémica), antes de realizar el diagnóstico de amiloidosis localizada.

El tratamiento tiene por objeto disminuir la producción de precursores, inhibir el depósito extracelular de amiloide e incrementar la lisis de los depósitos amiloideos existentes. Existen tratamientos específicos para cada tipo de amiloidosis. La combinación con prednisona/melfalán/ colchicina ha demostrado su eficacia. La colchicina ha demostrado ser eficaz para bloquear el depósito de amiloide, así como incrementar la supervivencia en la amiloidosis sistémica primaria $^{15}$.

Presentamos un caso de amiloidosis primaria localizada en glande peneano.

\section{CASO CLÍNICO}

Varón de 56 años, destacando entre sus antecedentes diabetes mellitus tipo II en tratamiento con insulina, intervenido de miopía y cataratas.

Acude a nuestra consulta por presentar craurosis prepucial que impide retracción de prepucio. A la exploración se palpa debajo de prepucio a nivel del glande un nódulo de unos tres centímetros no doloroso y muy consistente.

Con la sospecha de carcinoma de pene, se realiza con anestesia local incisión prepucial encontrándonos un nódulo de cuatro centímetros de diámetro, de color rojo amarillento, algo rugoso, consistente al tacto y situado en cara lateral de glande (Figs. 1 y 2). Se tomó una cuña profunda
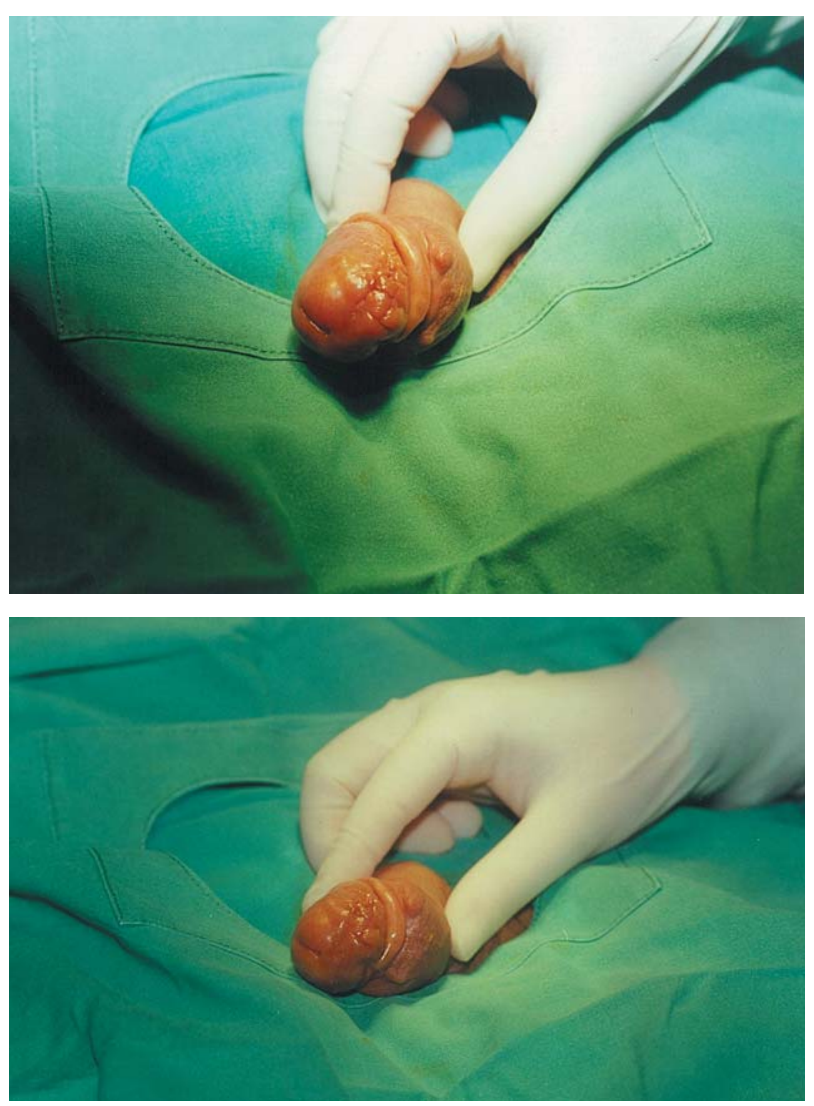

FIGURAS 1 y 2. Amiloidosis primaria localizada en glande peneano.

del nódulo y se envió para estudio anatomopatológico. Se decidió no realizar circuncisión ni exéresis del nódulo a la espera del resultado. Aunque con la incisión prepucial el glande pudo ser exteriorizado y realizar una higiene adecuada.

$\mathrm{El}$ resultado histológico es informado como amiloidosis nodular. Es curioso señalar que en el tiempo transcurrido a la espera del resultado histológico, al poder realizar una higiene adecuada, el aspecto del nódulo mejoró.

De forma reglada realizamos circuncisión y exéresis completa del nódulo, confirmando este segundo estudio histológico que se trataba de nódulo amiloidótico. Se realizó tinción con permanganato potásico que permitió diferenciar el amiloide tipo AA del AL, éste último característico de la forma primaria y no sensible al permanganato. La muestra tisular se tiñó con Rojo Congo y se examinó con luz polarizada observándose la típica birrefrigencia verde. Después de haber identificado el amiloide, se realizó estudio de proteínas e inmunohistoquímica. El compo- 
nente amiloide lo constituía el tipo AL, característico de la amiloidosis primaria y amiloidosis local. Este componente fibrilar se caracteriza por tener un depósito mayor de inmunoglobulinas Kappa o lambda.

Se realizó estudio complementario para descartar amiloidosis generalizada consistente en hemograma, bioquímica, proteinograma, PSA, Radiografía de tórax, ecografía abdominal y biopsia de mucosa rectal. El paciente no presentó síndrome nefrótico por lo que no creímos oportuno realizar biopsia renal. Tampoco presentaba artritis, insuficiencia cardíaca, hipotensión ni neuropatía. Todas las pruebas presentaron unos resultados normales por lo que se descartó amiloidosis generalizada.

Dos años después, la lesión no ha recidivado y el paciente no ha desarrollado amiloidosis generalizada.

\section{DISCUSIÓN}

La presencia de amiloidosis primaria de glande peneano es muy infrecuente. En la revisión bibliográfica realizada sólo hemos hallado 6 casos descritos en la literatura ${ }^{9-14}$. En todos los casos se trató de una lesión pequeña de 0,5 a 3 $\mathrm{cm}$ de diámetro, de color amarillento y consistencia elástica e indolora al tacto. Las lesiones se localizaron en cara lateral de glande ${ }^{9,10}$, surco balano prepucial ${ }^{12}$, meato ${ }^{13}$ y parameatal ${ }^{11,14}$.

La edad media es de 37 años (rango 20-67). Entre los antecedentes personales sólo destacaba un caso con gonorrea en la juventud ${ }^{14}$. En nuestro caso la edad era de 56 años, destacando como antecedente la diabetes mellitus.

Ninguno de los casos presentaba asociación con amiloidosis generalizada. Solamente dos casos presentaron nódulos amiloidóticos simultáneamente en otras zonas aunque localizados; uno en uretra ${ }^{14}$ y otro en traquea ${ }^{11}$.

En todos los casos el tratamiento fue la escisión local, salvo en un caso que se realizó radioterapia ${ }^{13}$.

La evolución fue favorable en casi todos los $\operatorname{casos}^{10,12-14}$. Sin embargo el caso que presentaba lesión traqueal ${ }^{11}$ presentó de forma sincrónica un seminoma testicular y tras linfadenectomía retroperitoneal falleció por sepsis, no encontrándose en la necropsia otros focos de amiloidosis.
Dado que el componente amiloide lo constituye el tipo AL, característico de la amiloidosis sistémica primaria y amiloidosis local, en ocasiones las biopsias tisulares (rectal, renal...) son importantes para establecer el diagnóstico diferencial. En nuestro caso se realizó biopsia rectal que fue normal y decidimos obviar la biopsia renal en ausencia de síndrome nefrótico.

En nuestro caso la amiloidosis pudo ocurrir como un proceso local favorecido por el factor irritativo que producía la falta de higiene por la craurosis prepucial, observándose una mejoría clínica tras mejorar este aspecto. Así mismo la posible influencia de la diabetes favoreció a nivel del glande procesos degenerativos e infecciosos. En algunos estudios se ha señalado como posible factor patogénico que la inflamación crónica de la mucosa puede llevar a producir inmunoglobulinas que por proteolisis sufren modificación y forman fibrillas insolubles que se depositan constituyendo el material amiloideo ${ }^{16,17}$.

La afectación del tracto genitourinario por amiloidosis primaria localizada no es en líneas generales muy frecuente. En una revisión sobre amiloidosis primaria localizada en tracto genitourinario de 84 casos recogidos de la literatura ${ }^{4}$ se encontró que 45 correspondían a vejiga, una cuarta parte al uréter o pelvis y el resto a la próstata, uretra y pene.

En conclusión, la amiloidosis primaria localizada en glande es una entidad muy infrecuente ante la que debemos hacer en un principio un diagnóstico diferencial con el cáncer de pene o el condiloma por su presentación como nódulo o lesiones purpúricas y petequias. Sólo el estudio histológico nos aportará el diagnóstico definitivo. El pronóstico es bueno cuando aparece en forma localizada pero siempre habrá que descartar enfermedad sistémica mediante historia clínica exhaustiva, antecedentes familiares, estudios analíticos y biopsias tisulares de otros órganos (recto, riñón, grasa subcutánea, túnel carpiano...) según la clínica de cada caso. En los casos que nos ocupan no fue preciso tratamiento con corticoides, melfalán o colchicina que vienen siendo la combinación estándar para el tratamiento de la forma sistémica, junto con el trasplante hepático y renal en sus formas graves. Sí que nos parece que la circuncisión en pacientes 
diabéticos y en casos de craurosis que impiden la correcta higiene pueden ser factores importantes para prevenir ente tipo de amiloidosis.

\section{REFERENCIAS}

1. Skinner M. Diagnosis of amyloidosis, in laboratory diagnostic procedures in the rheumatic diseases, 3d ed, AS Cohen (ed). Orlando, Fla, Grune \& Stratton, 1985.

2. Kyle RA, Greipp PR. Amyloidosis (AL): Clinical and laboratory features in 229 cases. Mayo Clin Proc. 1983;58(10): 665-683.

3. Husby G, Sletten K. Chemical and clinical classification of amyloidosis, 1985. Scand J Immunol. 1986;23(3):253-265.

4. Fujihara S, Glenner GG. Primary localized amyloidosis of the genitourinary tract. Immunohistochemical study on eleven cases. Lab Investig. 1981;44(1):55-60.

5. Esslimani M, Serre I, Granier M, Robert M, Baldet P, Costes V. Urogenital amyloidosis: clinico-pathological study of 8 cases. Ann Pathol. 1999;19(6):487-491.

6. Merrimen JL, Alkhudair WK, Gupta R. Localized amyloidosis of the urinary tract: case series of nine patients. Urology. 2006;67(5):904-909.

7. Fariña Perez LA, Ortiz Rey JA. Secondary bladder amyloidosis with severe recurrent hematuria: transurethral Mikuliz procedure as hemostatic option. Arch Esp Urol. 2005;58(7): 665-668.

8. Trivez Boned MA, Blas Marin M, García García MA, Gil Martínez P, García de Jalón Martínez A, Rioja Sanz LA Amiloidosis localizada de uretra Actas Urol Esp. 2002;26(1): 46-49.

9. Ritter M, Nawab RA, Tannenbaum M, Hakky SI, Morgan MB. Localized amyloidosis of the glans penis: a case report and literature review. J Cutan Pathol. 2003;30(1):37-40.
10. Srinivasan V, Kehinde EO, Harris MD, Turner AG. Localized amyloid of the glans penis. Br J Surg. 1992;79 (11): 1156 .

11. Weitzner S, Keen PE, Doughty WE. Primary localized amyloidosis of glans penis. Arch Dermatol. 1970;102(4):463464 .

12. Degos R, Cottenot F, Civatte J. Amyloïdose isolée du gland. Bull Soc Dermatol Syphiligr. 1961;68:159-160.

13. Friedmann E, Duperrat MB. Amyloïdose isolée du gland. Bull Soc Dermatol Syphiligr. 1967;74(3):313-314.

14. Bodner H, Retsky MI, Brown G. Primary amyloidosis of glands penis and urethra: resection and reconstruction. $\mathrm{J}$ Urol. 1981;125(4):586-588.

15. Zemer D, Pras M, Sohar E, Modan M, Cabili S, Gafni J. Colchicine in the prevention and treatment of the amyloidosis of familial mediterranean fever. $\mathrm{N}$ Engl $\mathrm{J}$ Med. 1986;17;314(16):1001-1005.

16. Ehara H, Deguchi T, Yanagihara M, Yokota T, Uchino F, Kawada Y. Primary localized amyloidosis of the bladder. An immunohistochemical study of a case. J Urol. 1992;147 (2): $458-460$

17. Fernandez H, Rovegno AR, Marrugar R, Lauterstein GA, Davalos Mitchel M, Lombardo M, et al. Amiloidosis ureteral primaria. Arch Esp Urol. 1998;51(5):485-490.

Correspondencia autor: Dra. M. Domínguez Domínguez. Servicio de Urología. Hospital de la Merced.

Avda. Constitución, 2. 41640 Osuna (Sevilla).

Tel.: 955077200

E-mail autor: montsedomin@hotmail.com

Información artículo: Nota Clínica

Trabajo recibido: octubre 2006

Trabajo aceptado: noviembre 2006 